\title{
Sonography of a case series of talar callosities
}

\author{
Loreto Luna-Bastante ${ }^{1}$ [ $\cdot$ Elena López Negrete ${ }^{2} \cdot$ Laura Alonso Naranjo $^{3}$ (D) Gastón Roustan ${ }^{2}$. \\ Fernando Alfageme ${ }^{2,4}$
}

Received: 9 December 2020 / Accepted: 25 January 2021 / Published online: 7 February 2022

(c) Società Italiana di Ultrasonologia in Medicina e Biologia (SIUMB) 2022

\begin{abstract}
Talar callosity is an uncommon condition characterized by asymptomatic hyperkeratotic plaques located on the dorsum of one or both feet. We have detected an increased number of children presenting with this condition after the COVID-19 lockdown. High-frequency ultrasound was performed in all patients who consulted because of talar callosity. All four cases shared similar ultrasound findings: thickening of the epidermis with effacement of the subepidermal low-echogenic band and diffuse thickening of the dermis and subcutaneous cellular tissue, with a diffuse decrease in echogenicity. The bone cortex was respected in all cases. No increase in vascularization was detected. Although it is a benign condition, it is important to recognize talar callosity to exclude worse conditions and to prevent unnecessary biopsies. To our knowledge, we are the first to describe ultrasonographic findings of talar callosity.
\end{abstract}

Keywords Talar callosity $\cdot$ Children $\cdot$ Dermatological ultrasound

\section{Introduction}

Talar callosity is an uncommon benign condition characterized by asymptomatic hyperkeratotic plaques located on the dorsum of one or both feet. The condition is related to some postures adopted when sitting on the floor [1], especially during some activities such as yoga [2] or some praying rituals [3-5]. Diagnosis is clinical, and a biopsy is rarely needed.

Loreto Luna-Bastante

loretoluna.abm@hotmail.com

Elena López Negrete

elopeznegrete@gmail.com

Laura Alonso Naranjo

Lauralunar13@gmail.com

Gastón Roustan

groustan@gmail.com

Fernando Alfageme

Feralfarol@yahoo.es

1 Department of Dermatology, Hospital Universitario Rey Juan Carlos, Madrid, Spain

2 Department of Dermatology, Hospital Universitario Puerta de Hierro, Madrid, Spain

3 Department of Dermatology, Complejo Hospitalario Universitario de Toledo, Toledo, Spain

4 Universidad Autónoma de Madrid, Madrid, Spain
The common histologic findings are compact orthokeratosis, hypergranulosis, and dermal papillary fibrosis [3]. To our knowledge, there is no previous ultrasonographic description of talar callosity.

Nowadays, almost all dermatological centres have highfrequency ultrasound equipment. Ultrasound complements clinical examination, dermoscopy, and biopsy in the initial differential diagnosis, not only in tumoural lesions $[6,7]$ but also in inflammatory lesions [8] and nail unit pathologies [9]. The aim of this paper is to provide a sonographic description of talar callosity.

\section{Case report}

Since June 2020, we have attended four Caucasian children (Figs. 1, 2, 3, 4) because of lesions affecting the dorsum of one or both feet, which had developed or worsened during the COVID-19 lockdown.

These lesions were hyperkeratotic, brownish, welldemarcated plaques located on the anterolateral aspect of one or both feet, coinciding with the prominence of the talar head or the navicular, lateral cuneiform, and cuboid bones. These locations correspond to the areas of friction and repeated trauma when children sit with crossed legs or with inappropriate postures on the floor. No pain, itching, 


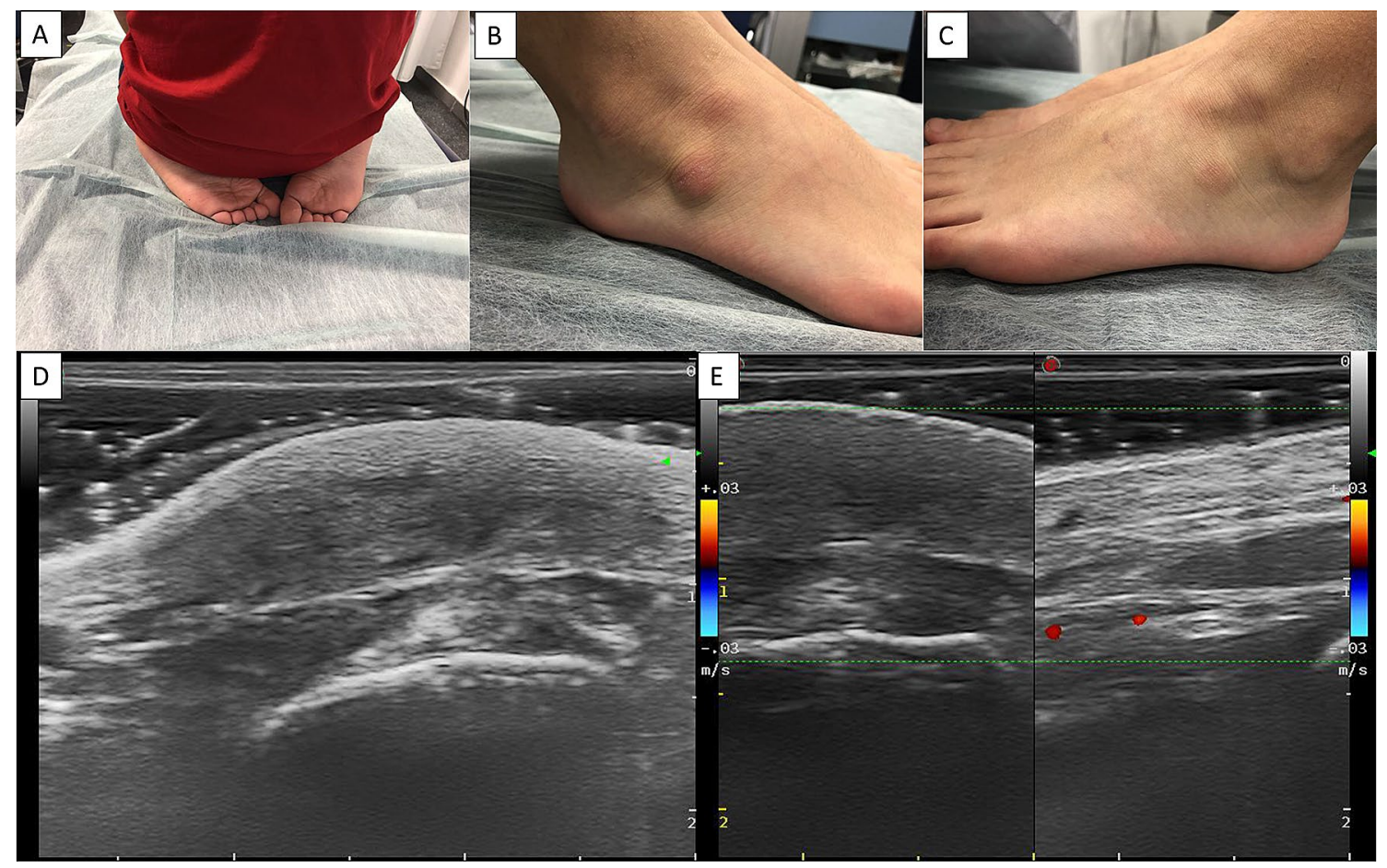

Fig. 1 Clinical and ultrasound images of Patient 1. Top row: a position adopted by the patient when sitting on the floor. Hyperkeratotic, pink to brown, well-demarcated plaque affecting the anterolateral aspect of $\mathbf{b}$ the right foot and $\mathbf{c}$ the left foot. Bottom row: $\mathbf{d}$ B mode

or burning was reported. The patients' characteristics are summarized in Table 1.

High-frequency ultrasound of the lesions was performed in all four patients (Figs. 1, 2, 3, 4) using a linear probe with a variable frequency of 6-18 $\mathrm{MHz}$ (Esaote MyLab Class C, Genova, Italy), in B mode and colour Doppler.

\section{Results}

The common findings were the following: thickening of the epidermis with effacement of the subepidermal low-echogenic band and diffuse thickening of the dermis and subcutaneous cellular tissue, with a diffuse decrease in echogenicity compared with healthy perilesional tissue. The bone cortex was respected in all cases. No increase in vascularization was detected by colour Doppler.

The subepidermal low-echogenic band (SLEB) consisted of a hypoechogenic band located immediately below the epidermis. The thickening of the SLEB has been related to photodamage, although the SLEB reflects not only structural alteration in the skin but also an extensive accumulation of water in the papillary dermis [10]. ultrasound of the affected area; we can observe diffuse thickening of the dermis and hypodermis compared with the non-affected skin, as shown in (e)

\section{Discussion}

Talar callosity, also known as callosity of crossed-legged sitting, is uncommon in occidental countries, but it is frequently seen in Arabic and Asian cultures, in relation to prayer [3-5], meditation [11], and other activities that involve crossed-legged sitting [1], such as yoga or even eating in some countries (e.g., India) [2]. Talar callosity has been previously described in children in relation to crossed-legged or kneeling positions on the floor [12]. The callosity can vary in its location, from the anterolateral aspect to the dorsum of the feet, depending on the adopted posture, and the location is usually away from common shoe contacts. Although talar callosities are usually bilateral, they tend to be more prominent on one foot [13].

Diagnosis is clinical, and a biopsy is rarely needed. The common histologic findings are compact orthokeratosis, hypergranulosis, and dermal papillary fibrosis. In contrast to lichen simplex chronicus, dermal fibrosis does not exhibit collagen bundles perpendicular to the epidermis [3].

The differential diagnosis consists of lichen simplex chronicus, eczema, psoriasis, neuropathy-related callosities, drug reactions, bone tumours, and other solid lesions 


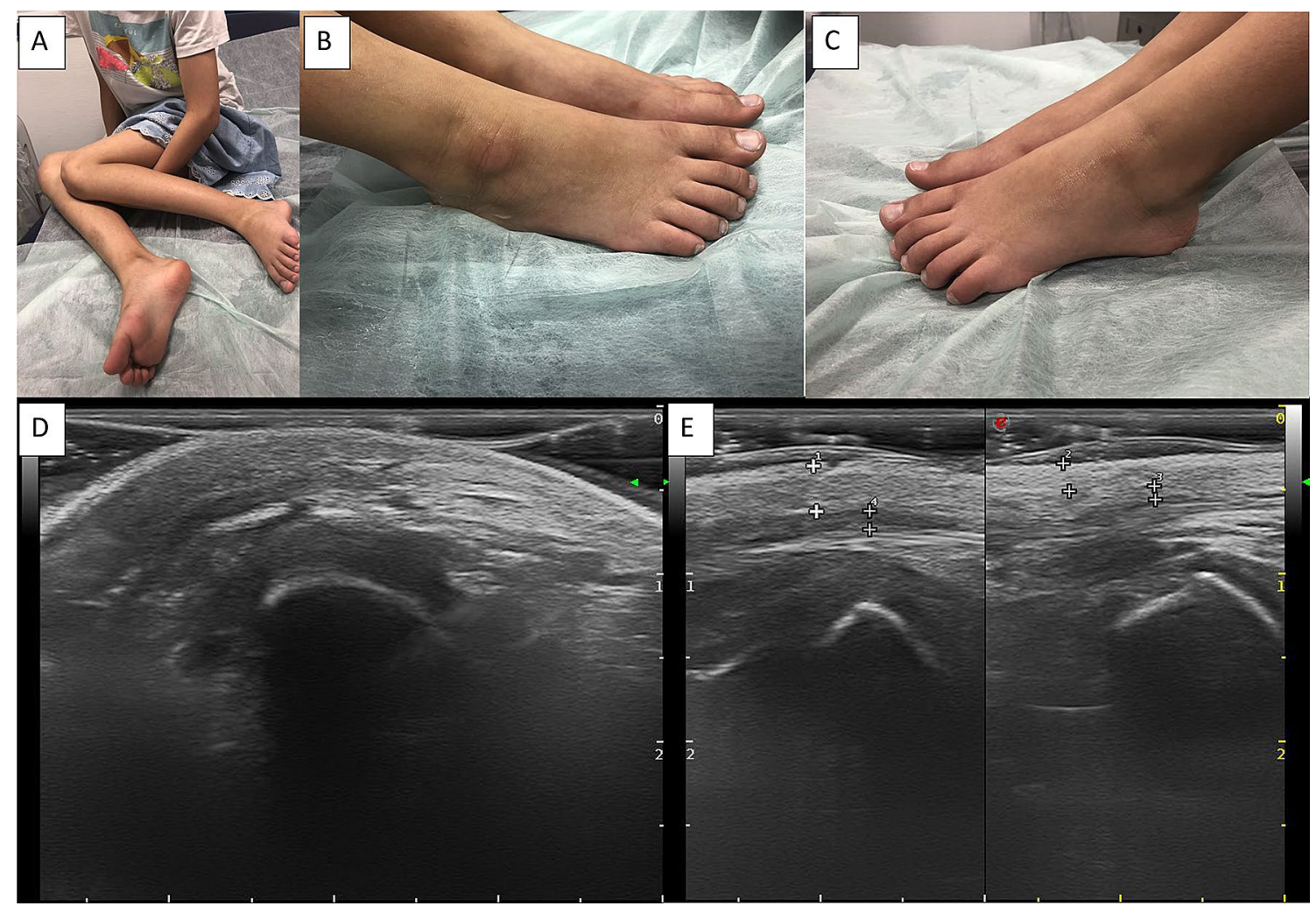

Fig. 2 Clinical and ultrasound images of Patient 2. Top row: a position adopted by the patient when sitting on the floor. $\mathbf{b}$ Hyperkeratotic, pink, well-demarcated plaque affecting the anterolateral aspect of the right foot. $\mathbf{c}$ The left foot is unaffected as it is not in contact with the ground. Bottom row: $\mathbf{d}$ B mode ultrasound of the affected area; we can observe effacement of the SLEB and diffuse thickening of the dermis and hypodermis compared with the non-affected skin, as shown in (e)
Fig. 3 Clinical and ultrasound images of Patient 3. Top row: a position adopted by the patient when kneeling on the floor. $\mathbf{b}$ Three hyperkeratotic, brownish, well-demarcated plaques affecting the anterolateral aspect of the left foot. Bottom row: c B mode ultrasound of the affected area, showing effacement of the SLEB and diffuse thickening of the dermis and hypodermis. $\mathbf{d}$ No increase in vascularization is seen with colour Doppler

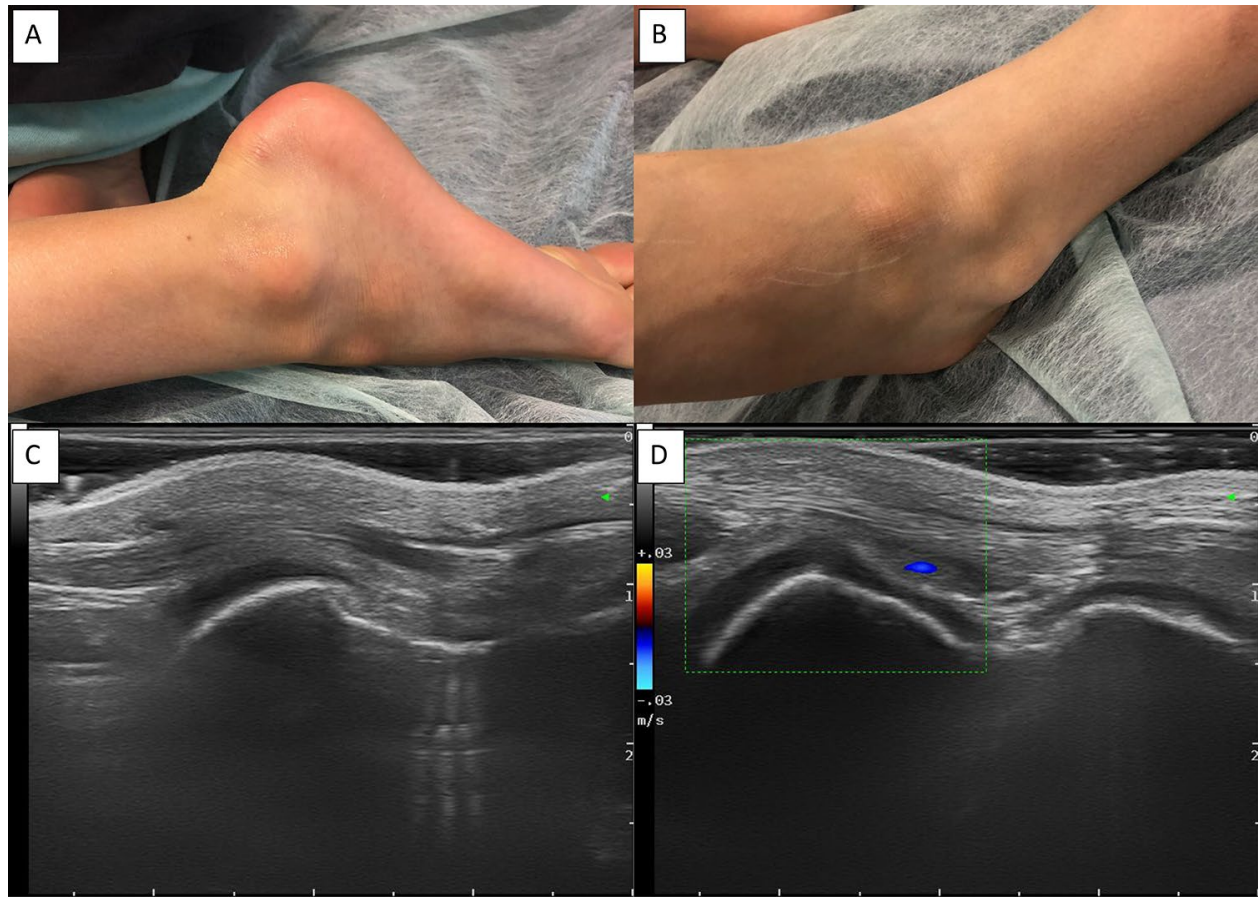


Fig. 4 Clinical and ultrasound images of Patient 4. Top row: a Position adopted by the patient when sitting crossed-legged on the floor. b Hyperkeratotic, brownish, well-demarcated plaque affecting the anterolateral aspect of the right foot. Bottom row: c B mode ultrasound of the affected area, showing effacement of the SLEB and diffuse thickening of the dermis and hypodermis. $\mathbf{d}$ No increase in vascularization is seen with colour Doppler

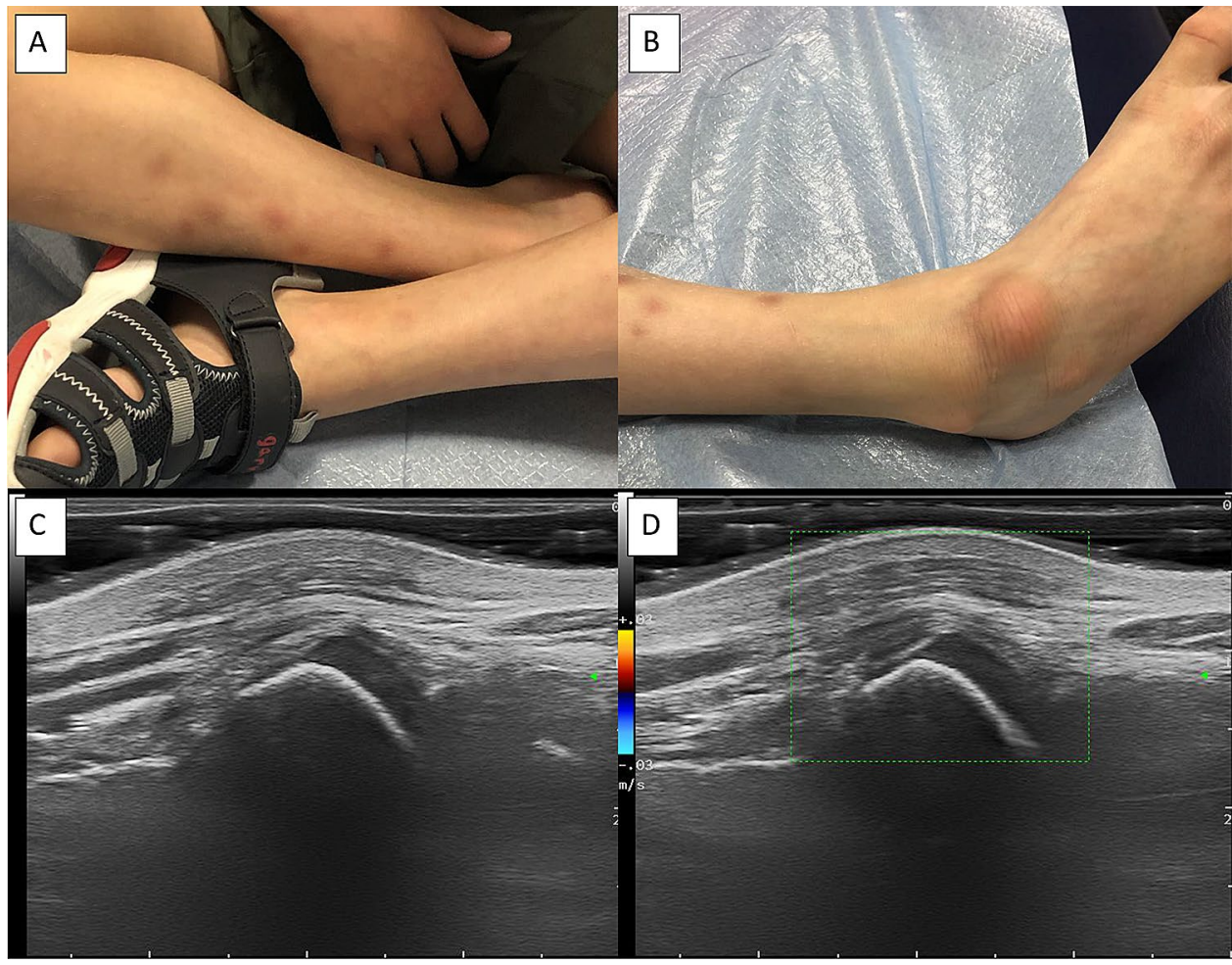

Table 1 Four patients which consulted because of talar callosity

\begin{tabular}{llllllll}
\hline & Age & Sex & Ethnicity & $\begin{array}{l}\text { Previous medi- } \\
\text { cal history }\end{array}$ & Unilateral or bilateral & $\begin{array}{l}\text { Lockdown child } \\
\text { favourite posture }\end{array}$ & Location \\
\hline Case 1 & 10 & Male & Caucasian & None & Bilateral & Kneeling & Anterolateral aspect of both feet \\
Case 2 & 8 & Female & Caucasian & None & Unilateral & Cross legs & Anterolateral aspect of right foot \\
Case 3 & 7 & Male & Caucasian & None & Unilateral & Kneeling & Anterolateral aspect of left foot \\
Case 4 & 8 & Male & Caucasian & None & Unilateral & Cross legs & Anterolateral aspect of right foot \\
\hline
\end{tabular}

that can stimulate reactive hyperkeratosis, among others [1, 2, 4, 5, 12-14].

To our knowledge, this is the first publication describing the diagnosing of talar callosity by imaging. In our opinion, it is an extremely useful non-invasive technique that allows the worst prognosis to be excluded and the parents of the affected children to be reassured.

Treatment options include topical keratolytic agents such as urea or salicylic acid, curettage, and surgical removal. However, talar callosity tends to recur if the causal posture persists.

Although it is difficult to demonstrate, the increase in consultations for talar callosity could, in our opinion, be related to the more sedentary lifestyle during the COVID19 confinement. In one international study in which 1,047 adults responded to a questionary, it was observed that the daily sitting time increased from five to eight hours per day [15]. The COVID-19 lockdown affected not only adult people. In Spain, schools, gymnasiums, and sport centres were closed, and children's daily activities depended on online educational and sporting resources. This implied a reduction in physical activity and an increase in children's sedentarism [16]. It would be interesting to perform future observational studies to demonstrate a correlation between sedentarism and talar callosity.

\section{Conclusion}

Although it is a well-defined, benign condition, it is important to recognize talar callosity to avoid incorrect diagnosis and to identify worse underlying conditions, such as tumours [14]. To our knowledge, we are the first to describe ultrasonographic findings of talar callosity. Ultrasonography can help to identify talar callosity, and it is useful in excluding underlying tumoural causes. 
Funding None.

\section{Compliance with ethical standards}

Conflict of interest Authors have no conflicts of interest to declare.

Ethical standards This work has been carried out in accordance with the requirements expressed in the Declaration of Helsinki (64th General Assembly, Fortaleza, Brazil, October 2013) as well as the current legislation in Spain regarding the performance of observational studies.

Consent for publication All patient-reported gave their consent for the publication of photographs. The reported patients have been evaluated and treated according to the usual clinical practice. They have not been subjected to any experimental protocol.

\section{References}

1. Omidian M, Omidian E (2011) Callosity of ankles among people with the habit of sitting with crossed legs: a cosmetic problem. Dermatol Rep 3:e24

2. Verma SB, Wollina U (2008) Callosities of cross legged sitting: "yoga sign"-an under-recognized cultural cutaneous presentation. Int J Dermatol 47:1212-1214

3. Abanmi AA, Al Zouman AY, Al Hussaini H, Al-Asmari A (2002) Prayer marks. Int J Dermatol 41:411-414

4. Fosse N, Rast AC, Kammermann A, Sonderegger J, Navarini A, Goldust M et al (2020) Pitfall prayer marks: recognition and appropriate treatment: a case report and review of literature. DermatolTher 33:e13176

5. Saif I, Woywodt A (2009) Talar callosity ('prayer foot') in a haemodialysis patient. NDT Plus 2:89-90
6. Catalano O, Roldán FA, Varelli C, Bard R, Corvino A, Wortsman X (2019) Skin cancer: findings and role of high-resolution ultrasound. J Ultrasound 22:423-431

7. Catalano O, Varelli C, Sbordone C, Corvino A, De Rosa D, Vallone $G$ et al (2020) A bump: what to do next? Ultrasound imaging of superficial soft-tissue palpable lesions. J Ultrasound 23:287-300

8. Vergara-de-la-Campa L, Cembrero-Saralegui H, Luna-Bastante L, Martínez-Lorenzo ER, Alfageme-Roldán F. Usefulness of ultrasound for treatment and follow-up of cutaneous leishmaniasis. J Ultrasound. 2020 [cited 2021 Jan 2]; Available from https://doi. org/10.1007/s40477-020-00540-0

9. Aluja Jaramillo F, QuiasúaMejía DC, MartínezOrdúz HM, González AC (2017) Nail unit ultrasound: a complete guide of the nail diseases. J Ultrasound 20:181-192

10. Gniadecka M (2001) Effects of ageing on dermal echogenicity. Skin Res Technol 7:204-207

11. Ur Rehman H, Asfour NA (2010) Prayer nodules. CMAJ Can MedAssoc J 182:E19

12. Lim KBL, Tawng DK, Lokino ES, Raveendran R, Tay GT (2011) Talar callosity in children-what, who, why? J Pediatr 158:340

13. Verbov JL, Monk CJ (1991) Talar callosity-a little-recognized common entity. ClinExpDermatol 16:118-120

14. Keser S, Bayar A (2005) Osteochondroma of the talar neck: a rare cause of callosity of the foot dorsum. J Am Podiatr Med Assoc 95:295-297

15. Ammar A, Brach M, Trabelsi K, Chtourou H, Boukhris O, Masmoudi L et al (2020) Effects of COVID-19 home confinement on eating behaviour and physical activity: results of the ECLBCOVID19 international online survey. Nutrients 12(6):1583

16. Shahidi SH, Stewart Williams J, Hassani F (2020) Physical activity during COVID-19 quarantine. Acta Paediatr 109(10):2147-2148

Publisher's Note Springer Nature remains neutral with regard to jurisdictional claims in published maps and institutional affiliations. 